\title{
Diameter of Superior Rectal Vein - CT Predictor of KRAS Mutation in Rectal Carcinoma
}

This article was published in the following Dove Press journal:

Cancer Management and Research

\author{
Chenyu Song ${ }^{l, *}$ \\ Bingqi Shen ${ }^{1, *}$ \\ Zhi Dong',* \\ Zhenzhen Fan ${ }^{2}$ \\ Ling $\mathrm{Xu}^{3}$ \\ Zi-Ping $\mathrm{Li}^{\mathbf{1}}$ \\ Yin $\mathrm{Li}^{4}$ \\ Shi-Ting Feng \\ 'Department of Radiology, The First \\ Affiliated Hospital, Sun Yat-Sen \\ University, Guangzhou, Guangdong \\ 510080, People's Republic of China; \\ ${ }^{2}$ Department of Radiology, Luoyang \\ Central Hospital Affiliated to Zhengzhou \\ University, Luoyang, Henan 47I009, \\ People's Republic of China; ${ }^{3}$ Faculty of \\ Medicine and Dentistry, University of \\ Western Australia, Perth, Australia; \\ ${ }^{4}$ Department of Gastroenterology \\ Surgery, The First Affiliated Hospital, Sun \\ Yat-Sen University, Guangzhou, \\ Guangdong, 510080, People's Republic of \\ China \\ *These authors contributed equally to \\ this work
}

Correspondence: Shi-Ting Feng

Department of Radiology, The First Affiliated Hospital, Sun Yat-Sen University, 58th, the

Second Zhongshan Road, Guangzhou,

Guangdong, People's Republic of China

Tel +86 20-87755766 Extension 847I

Fax +86 20-87615805

Email fengsht@mail.sysu.edu.cn

Yin $\mathrm{Li}$

Department of Gastrointestinal Surgery, The First Affiliated Hospital, Sun Yat-Sen

University, Guangzhou, Guangdong 510080 ,

People's Republic of China

Email liyin2736@I63.com
Background: The purpose of this study was to investigate the feasibility of CT parameters to predict the presence of KRAS mutations in rectal cancer patients. The relationship between the presence of a KRAS mutation and pathological findings was evaluated simultaneously.

Methods: Eighty-nine patients (29 females, 60 males, age 27-90, mean 59.7 \pm 12 years) with pathologically proven rectal cancer were enrolled. A KRAS mutation test was completed following surgery. Parameters evaluated on CT included the tumor location, the diameter of the superior rectal vein (SRV) and inferior mesenteric vein (IMV), the presence of calcification, ulceration, lymph node enlargement (LNE), distant metastasis, tumor shape (intraluminal polypoid mass, infiltrative mass, or bulky), circumferential extent ( $\mathrm{C} 0-\mathrm{C} 1 / 4, \mathrm{C} 1 / 4-\mathrm{C} 1 / 2$, $\mathrm{C} 1 / 2-\mathrm{C} 3 / 4$, or $\mathrm{C} 3 / 4-\mathrm{C} 1$ ), enhanced pattern (homogeneous or heterogeneous), $\mathrm{CT}$ ratio, and the length of the tumor (LOT). Pathological findings included lymphovascular emboli, signet ring cell, peripheral fat interval infiltration, focal ulcer, lymph node metastasis, tumor pathological type, and differentiation extent. The correlations between KRAS status and CT parameters, and KRAS status and pathological findings were investigated. The accuracy of CT characteristics for predicting KRAS mutation was evaluated.

Results: A KRAS mutation was detected in 42 cases. On CT image, the diameter of the SRV was significantly increased in the KRAS mutation group compared to in the KRAS wild-type group ( $4.6 \pm 0.9 \mathrm{~mm}$ vs $4.2 \pm 0.9 \mathrm{~mm}, p=0.02$ ), and LNE was more likely to occur in the KRAS mutation group $(73.3 \%$ vs $26.7 \%, p=0.03)$. There was no significant difference between the KRAS mutation group and the KRAS wild-type group on the other CT parameters (location, IMV, calcification, ulcer, distant metastasis, tumor shape, enhanced pattern, circumferential extent, CT ratio, and LOT). In the pathological findings, a KRAS mutation was more likely to occur in the middle differentiation group $(p=0.03)$. No significant difference was found between the KRAS mutation group and the KRAS wild-type group in the presence of lymphovascular emboli, signet ring cell, peripheral fat interval infiltration, focal ulcer, lymph node metastasis, and tumor pathological type. With the best cut-off value of $4.07 \mathrm{~mm}$, the AUC of the SRV to predict a KRAS mutation was 0.63 with a sensitivity of $76.2 \%$ and a specificity of $48.9 \%$.

Conclusion: It was feasible to use the diameter of the SRV to predict a KRAS mutation in rectal cancer patients, and LNE also can be regarded as an important clue on preoperative CT images.

Keywords: rectal neoplasms, mutation, computed tomography, superior rectal vein

\section{Background}

Colorectal cancer (CRC) is the third most commonly diagnosed malignancy and the fourth leading cause of cancer-related deaths in the world. ${ }^{1}$ Rectal carcinoma 
accounts for around $30-35 \%$ of all CRC cases. ${ }^{2}$ In China, the incidence of $\mathrm{CRC}$ was approximately 37.6 per 100,000 , with a mortality of 19.1 per $100,000 .^{3}$ From 1972 to 2005, the rates of rectal cancer increased from 7.68 and 6.51 to 11.45 and 8.28 per 100,000 in males and females, respectively. ${ }^{4}$ Nevertheless, with the improvement of therapeutic regimens, decreasing rectal cancer mortality has also been observed. ${ }^{5}$ Anti-EGFR (epidermal growth factor receptor) drugs are proven effective for rectal cancer. However, tumor response to anti-EGFR drugs is significantly related to the presence of a KRAS (Kirsten rat sarcoma viral oncogene homologue) mutation. Generally, metastatic CRC patients with a KRAS mutation tend to be resistant to anti-EGFR therapy. ${ }^{6}$ According to the American Society of Clinical Oncology, if a KRAS mutation in codon 12 or 13 is detected, then patients with metastatic colorectal carcinoma should not receive antiEGFR antibody therapy as part of their treatment. ${ }^{7}$ For this reason, KRAS mutation evaluation shows great importance for rectal cancer patients before pharmaceutical treatment is commenced.

Commonly, KRAS status (mutated type or wild type) is tested in postoperative pathology specimens. However, patients with advanced or metastatic rectal carcinoma may not receive surgery treatment or obtain specimens for testing. Biopsy through colonoscopy is one of the main invasive methods of specimen acquisition for KRAS status testing. The accuracy is influenced by sampling error and tumor heterogeneity. According to Jahn et al, different metastatic lymph nodes can be segregated into multiple intra-tumoral KRAS mutations. ${ }^{8}$ And despite clinical success in gene testing, the potential loss of gene information during the DNA extraction process is still an unavoidable factor.

As a noninvasive method, medical imaging could be adapted for the further assessment of neoplastic features. Though MRI (magnetic resonance imaging) has been regarded as the first choice of local staging in rectal carcinoma, for patients with MRI contraindications, contrast enhanced CT also presents great value for diagnosis. There has been an increasing amount of studies exploring the correlation between cancer biological markers and imaging findings. A few studies have focused on predicting KRAS mutations through various imaging methods such as PET-CT (positron emission tomography-computed tomography) $)^{9,10}$ and MRI. ${ }^{11}$ However, little research has been performed on predicting KRAS mutations in rectal carcinoma by preoperative MSCT (multi-slice computed tomography) examination. Our study set out to assess the effect of CT parameters to predict a KRAS mutation in patients with rectal carcinoma, including observed CT features and anatomical-based measurement. Meanwhile, the SRV (superior rectal vein) only receives the blood flow from the rectum, making the SRV particularly sensitive to any factors influencing the biological process of the rectum. And the SRV has been proven to be significantly increased in rectal cancer with lymphovascular invasion. ${ }^{12}$ In our study, CT parameters, especially the SRV, have been assessed to predict KRAS mutations. Lymph node enlargement (LNE), distant metastasis, enhanced pattern, CT ratio, and the length of tumor were supposed to imply the different invasive behaviors of rectal carcinoma. ${ }^{5}$ The correlation between pathological findings and KRAS status has also been evaluated.

\section{Methods}

\section{Patients}

This study was conducted in accordance with the ethical guidelines for human research and received Institutional Review Board or ethical committee approval. Written informed consent was obtained from all patients in the study.

The inclusion criteria are as follows: 1) preoperative CT data; 2) no preoperative chemotherapy or radiation therapy; 3) surgical treatment commenced within 1 week after CT examination; 4) pathological proven rectal carcinoma; and 5) KRAS mutation testing was performed after surgery. The exclusion criteria are as follows: 1) presence of other malignant, infectious, or vascular disease in the abdominopelvic area instead of rectal carcinoma; and 2) poor imaging quality for measuring the $\mathrm{CT}$ predictors.

A total of 285 patients with surgically proven rectal carcinoma from December 2015 to October 2017 in the First Affiliated Hospital of Sun Yat-Sen University were enrolled in this retrospective study. One hundred and eighty-seven patients without KRAS mutation evaluation, three patients with incomplete $\mathrm{CT}$ images, and six patients with other malignant tumors were excluded (Figure 1).

\section{CT Protocol}

All patients underwent enema preparation and fasting $6 \sim 8$ hours before the CT scan. To expand the intestine carefully, patients were given $1.6 \sim 2.0 \mathrm{~L}$ of $2.5 \%$ isotonic mannitol solution 1 hour before the CT scan; $0.4 \sim 0.5 \mathrm{~L}$ of $2.5 \%$ isotonic mannose at $45 \mathrm{~min}, 30 \mathrm{~min}$, 
From 2015.12 to 2017.10

The First Affiliated Hospital, Sun Yat-sen University

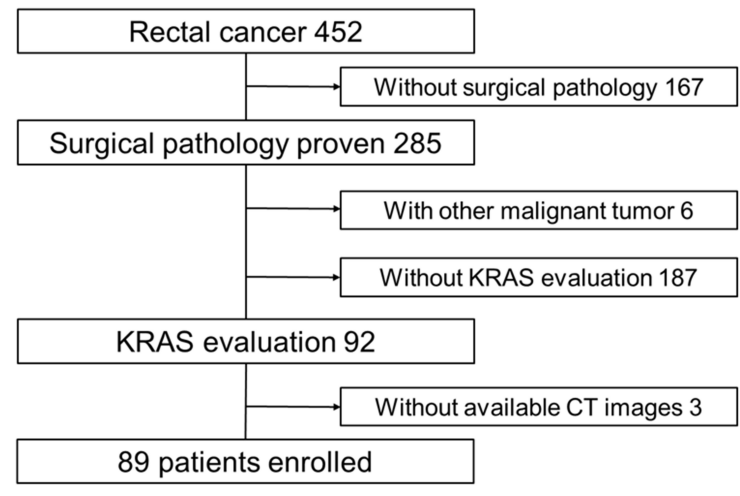

Figure I Data filtering process

and $15 \mathrm{~min}$ before the $\mathrm{CT}$ scan; and $300 \sim 500 \mathrm{~mL}$ of a saline enema immediately before the CT scan.

All patients were scanned using a 320-detector row CT machine (Aquilion ONE; Toshiba Medical System, Tokyo, Japan), with the same scanning parameters as follows: tube voltage, $120 \mathrm{kV}$; tube current, $250 \mathrm{~mA}$; standard value, $320 \times 0.5 \mathrm{~mm}$; slice thickness, $1 \mathrm{~mm}$; slice gap, $1 \mathrm{~mm}$. All patients were in a supine position, with a CT scan range from the top of the diaphragm to the level of the ischial tubercle. Iopromide (Ultravist 300; Schering, Berlin, Germany) was selected as the contrast agent with an injection flow rate of $3.5 \mathrm{~mL} / \mathrm{s}$. Unenhanced and enhanced CT scans were taken at $32 \mathrm{~s}$ and $60 \mathrm{~s}$ after injecting iopromide, respectively. All data were later delivered to the workstation (Vitrea2; Toshiba Medical System, Tokyo, Japan) for processing.

\section{CT Features}

The preoperative $\mathrm{CT}$ data of all patients were interpreted independently by two diagnostic radiologists with 15 and 25 years of experience, respectively, who were blinded to the patients' pathological diagnosis. The diameter of the SRV and IMV (inferior mesenteric vein) was measured on preoperative CT images during the venous phase. (The minimum diameter of the relevant level was measured.) The diameter of the SRV was measured on the second sacral vertebral plane (better differentiation of the SRV from surrounding adipose tissue due to its position in the transverse plane at this level). The diameter of the IMV was measured at $5 \mathrm{~mm}$ from its root into the superior mesenteric vein or splenic vein (Figures 2 and 3). The enhanced ratio (ER, the ratio of $\mathrm{CT}$ value between lesion and aorta or branch artery at the same plane) and the

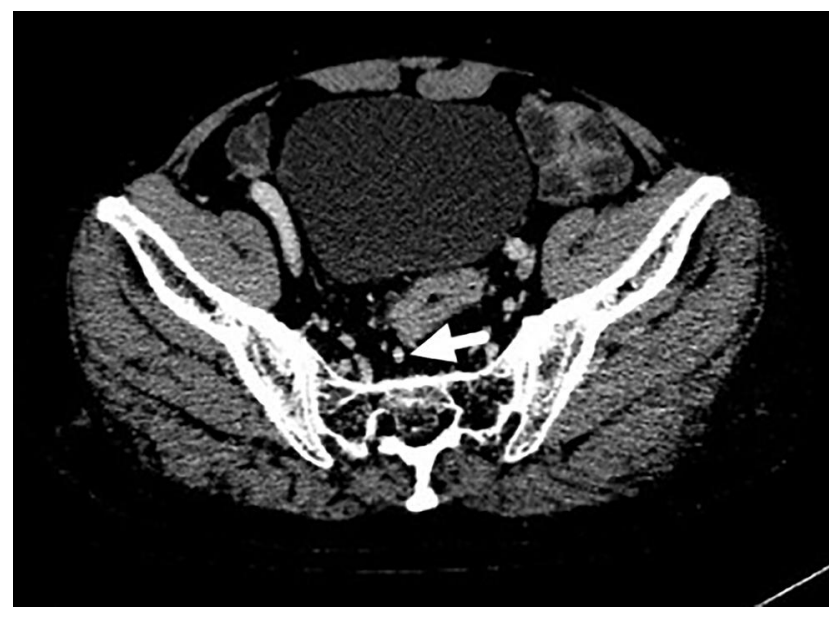

Figure 2 Position of the SRV is shown with the white arrow on the second sacral vertebral transverse plane. The white line represents the diameter of the SRV.

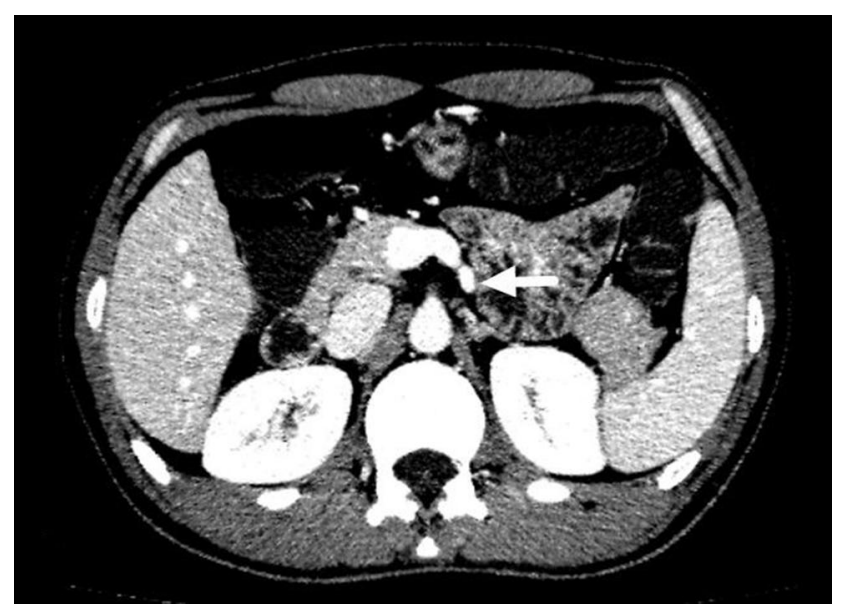

Figure 3 Position of the IMV is shown with the white arrow.

length of the tumor (LOT, length on the sagittal plane) were measured during the venous phase. The final data above were taken as the mean value of the two specialists' readings. The presence of calcification and ulcer was observed on the unenhanced phase and venous phase, respectively. The lesions were classified as high (10.1-15 $\mathrm{cm}$ from the anal verge), middle $(5.1-10 \mathrm{~cm}$ from the anal verge), and low $(0-5 \mathrm{~cm}$ from the anal verge) tumors according to the location. Tumor shape was divided into three types: intraluminal polypoid mass, infiltrative mass, and bulky. The circumferential extent of the tumor was divided into four types on the axial bowel plane: $\mathrm{C} 0-\mathrm{C} 1 / 4, \mathrm{C} 1 / 4-\mathrm{C} 1 / 2, \mathrm{C} 1 / 2-\mathrm{C} 3 / 4$, and $\mathrm{C} 3 / 4-\mathrm{C} 1$. Tumor density was observed on unenhanced and enhanced phases, respectively. Unclear boundaries of serosa mean increased density of the fat interval beside the tumor. The 
definition of lymph node enlargement is a short axis of the lymph node more than $8 \mathrm{~mm} .{ }^{13}$ The lymph node density is also observed on the venous phase. The presence of distant metastases refers to carcinoma lesions present in other distant organs or tissues. If two radiologists had any disagreement on the above observed CT features, the debating feature would be reread until a consensus is reached.

\section{Pathological Data and KRAS Testing}

The presence of lymph node metastasis, signet ring cell, lymphovascular emboli, focal ulcer, and tumor differentiation degree were all confirmed on pathological examination. Peripheral fat interval infiltration refers to the rectal cancer cell breaking into the perirectal fat interval. Tumor pathological types include adenocarcinoma, mucinous adenocarcinoma, and mixed type with good, moderate, and poor differentiation in three differentiation extents. The TNM staging followed the American Joint Committee on Cancer for colorectal cancer, 8 th edition. ${ }^{14}$

KRAS mutation evaluation was carried out using DNA extracted from paraffin sections (pathological tissue acquired after surgery). Mutational analysis for KRAS was performed using the CFX96 Real-Time PCR Detection System (Bio-Rad, Philadelphia, PA, USA) with the PNA clamp ${ }^{\mathrm{TM}}$ KRAS mutation detection kit (Panagene, Inc., Daejeon, Korea). Codon 12 and codon 13 were detected.

\section{Statistical Analysis}

The analyses were performed using SPSS version 25 and MedCalc Version 19.4.1. The consistency check was conducted between the data measured by two radiologists. Two independent-sample $t$-tests were used in continuous variables between the KRAS mutation and KRAS wildtype groups when continuous variables were consistent with a normal distribution. When continuous variables were not consistent with a normal distribution, nonparametric $t$-tests were used in these continuous variables. The difference between the KRAS mutation and KRAS wildtype groups was analyzed using a chi-squared test for categorical variables, and Fisher's exact test was applied when categorical variables were not consistent with the conditions of the chi-squared test. Difference with $p<0.05$ was considered as statistically significant. Binary logistics regression was used to select variables, and the receiver operating characteristic (ROC) curve was used to evaluate the effectiveness of diagnosis through the area under the curve (AUC).

\section{Results}

\section{Patient Characteristics}

A total of 89 patients (29 females, 60 males, age 27-90, mean $59.7 \pm 12$ years) were enrolled in accordance with the above inclusion and exclusion criteria. In $\mathrm{T}$ stage, there were 14 patients with T2 stage, 27 patients with T3 stage, 46 patients with T4 stage, and two patients with an unevaluable primary tumor. In $\mathrm{N}$ stage, there were 51 patients with N0 stage, 23 patients with N1 stage, 13 patients with $\mathrm{N} 2$ stage, and two patients with unevaluable regional lymph nodes. In M stage, there were six patients with M1.

\section{CT Parameters and KRAS Mutation}

The data measured by two radiologists showed good consistency. The cronbach's alpha coefficient was 0.998 for the SRV between two radiologists. We use logistic regression to select variables to predict KRAS mutation and the SRV diameter was the only selected variable. The diameter of the SRV was significantly increased in the KRAS mutation group compared to the KRAS wild-type group (4.62 $\pm 0.94 \mathrm{~mm}$ vs $4.19 \pm 0.82 \mathrm{~mm}, p=0.02$ ). With the best cutoff value of $4.07 \mathrm{~mm}$, the sensitivity and specificity were $76.2 \%$ and $48.9 \%$, respectively. The positive and negative predictive values were $57.1 \%$ and $69.7 \%$. LNE was more likely to occur in the KRAS mutation group $(73.3 \%$ vs $26.7 \%, p=0.03$; Figures 4 and 5). No significant difference was found between the KRAS mutation group and the KRAS wild-type group on the other CT parameters (location, the diameter of IMV, calcification, ulcer, distant metastasis, tumor shape, circumferential extent, enhanced pattern, CT ratio, and LOT) (Tables 1 and 2).

\section{Pathological Findings and KRAS Mutation Status}

In pathological findings, KRAS mutations were more likely to occur in the middle differentiation group $(p=0.03)$. No significant difference was found between the KRAS mutation group and the KRAS wild-type group in the presence of lymphovascular emboli, signet ring cell, peripheral fat interval infiltration, focal ulcer, lymph node metastasis, and tumor pathological type (Table 3).

\section{Discussion}

Over the past two decades, the specific clinical significance of KRAS mutation for patients' individual medical management has been studied and applied to patient 


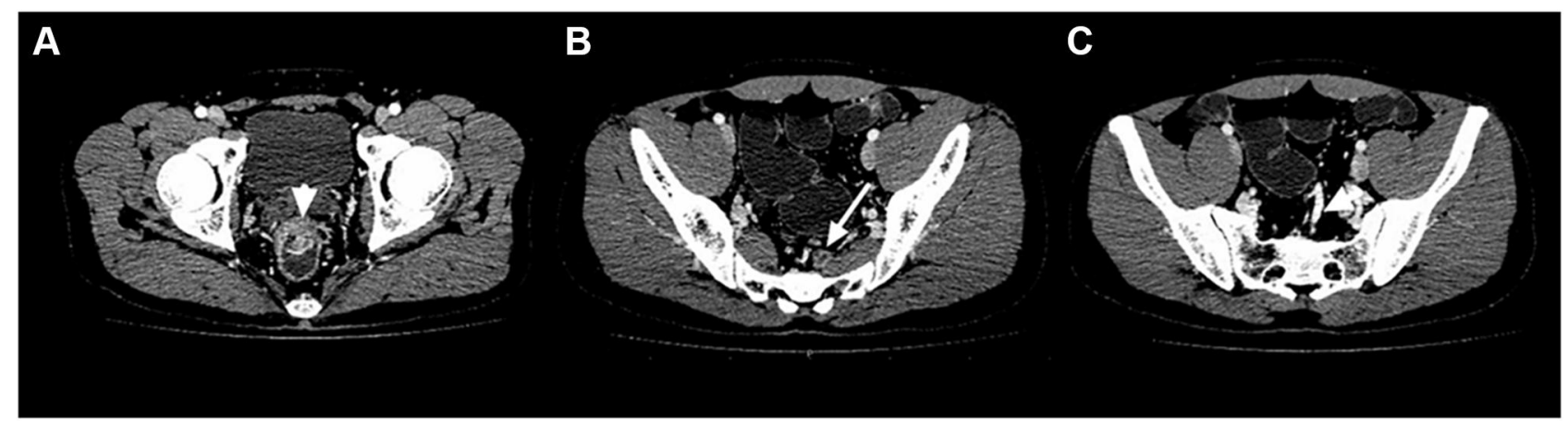

Figure 4 Transverse MSCT of a 40-year-old male patient with rectal cancer with a KRAS mutation: rectal carcinoma lesion (A, white arrow with short tail) was on the anterior rectal wall with LNE (B, white arrow with long tail). The short axis length of the enlarged lymph node was $12 \mathrm{~mm}$. The diameter of the SRV (C, white arrow with short tail) was dilated $(5.0 \mathrm{~mm},>4.5 \mathrm{~mm})$.

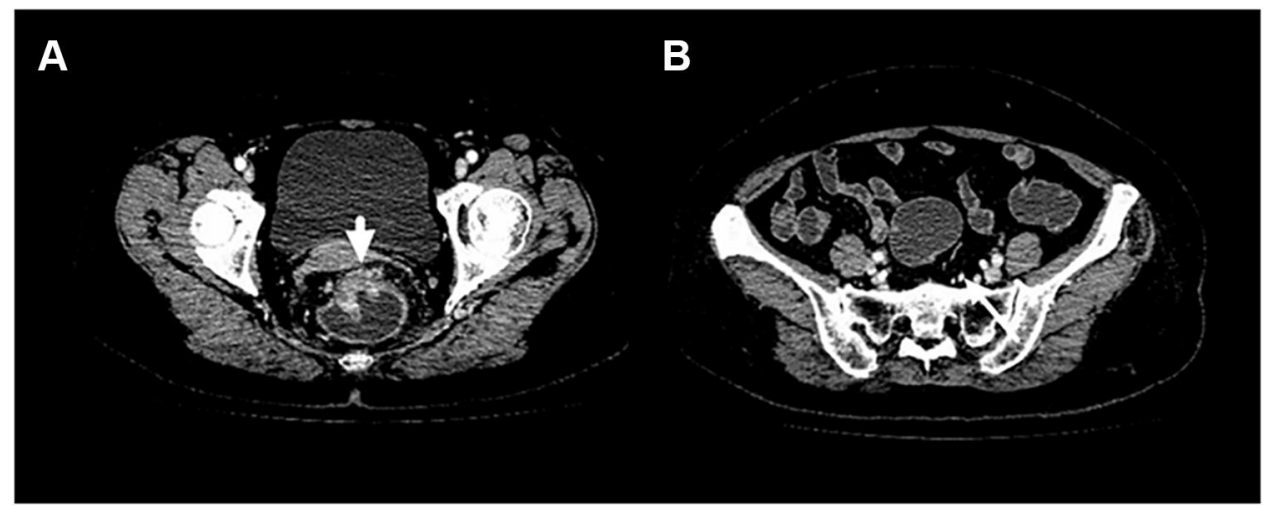

Figure 5 Transverse MSCT of a 64-year-old female patient with rectal cancer without a KRAS mutation: rectal carcinoma lesion (A, white arrow with short tail) was on the anterior rectal wall without LNE. The diameter of the SRV (B, white arrow with long tail) was normal size $(3.5 \mathrm{~mm})$.

management. ${ }^{15}$ There is a consensus that anti-EGFR antibody therapy is an effective choice for some metastatic rectal carcinoma patients without a KRAS mutation. ${ }^{16}$ The reasons why patients cannot benefit from anti-EGFR therapy may be explained by the heterogeneity of the tumor. However, medical imaging methods may disclose certain features associated with KRAS mutations. The feasibility of combining medical imaging parameters to predict KRAS mutations has been proven in some previous studies. ${ }^{17-19}$ In the study by Lovinfosse et $\mathrm{al},{ }^{20}$ rectal cancers with KRAS or NRAS mutations displayed a significantly higher glucose metabolism than wild-type cancers with ${ }^{18}$ F-FDG PET/CT imaging. Kawada et al ${ }^{9,21}$ found that ${ }^{18} \mathrm{~F}$-FDG accumulation into metastatic CRC assessed by SUVmax (the maximum standardized uptake value for the primary tumor) was associated with KRAS status. As for MRI, Xu et $\mathrm{al}^{22}$ found lower mean ADC (apparent diffusion coefficient) and higher $\mathrm{D}^{*}$ (pseudo diffusion coefficient) values on MRI with KRAS mutations in rectal carcinoma. And the study by Shin et $\mathrm{al},{ }^{5}$

Table I CT Predictors and KRAS Status (continuous variables)

\begin{tabular}{|c|c|c|c|c|c|}
\hline & \multirow[t]{2}{*}{ Test of Normality } & \multicolumn{2}{|l|}{ KRAS Status } & \multirow[t]{2}{*}{$\mathbf{t} / \mathbf{z}$} & \multirow[t]{2}{*}{$p$} \\
\hline & & Mutated $(n=42)$ & Wild $(n=47)$ & & \\
\hline Length $(\mathrm{mm})$ & 0.20 & $43.49 \pm 16.35$ & $45.16 \pm 13.13$ & 0.53 & 0.59 \\
\hline $\mathrm{SRV}(\mathrm{mm})$ & 0.10 & $4.62 \pm 0.94$ & $4.19 \pm 0.82$ & -2.30 & 0.02 \\
\hline IMV (mm) & 0.02 & $4.90(4.47,5.47)$ & $4.98(4.42,5.54)$ & -0.25 & 0.80 \\
\hline CT ratio (unenhanced) & 0.01 & $0.89(0.79,1.01)$ & $0.94(0.76,1.12)$ & -0.65 & 0.52 \\
\hline CT ratio (venous phase) & 0.20 & $0.52 \pm 0.08$ & $0.55 \pm 0.09$ & 1.58 & 0.12 \\
\hline
\end{tabular}


Table 2 CT Predictors and KRAS Status (categorical variables)

\begin{tabular}{|c|c|c|c|c|c|}
\hline & \multicolumn{2}{|c|}{ KRAS Status } & \multirow[t]{2}{*}{ Total } & \multirow[t]{2}{*}{$\mathbf{t} / \chi^{2}$} & \multirow[t]{2}{*}{$p$} \\
\hline & Mutated & Wild & & & \\
\hline Sex & & & & 1.10 & 0.29 \\
\hline M & $26(28.3)$ & $34(31.7)$ & $60(60.0)$ & & \\
\hline $\mathrm{F}$ & 16 (13.7) & $13(15.3)$ & $29(29.0)$ & & \\
\hline Calcification & & & & - & 0.46 \\
\hline Y & $2(1.4)$ & I (I.6) & $3(3.0)$ & & \\
\hline$N$ & $40(40.6)$ & $46(45.4)$ & $86(86.0)$ & & \\
\hline Ulcer & & & & 0.47 & 0.50 \\
\hline Y & 37 (35.9) & $39(40.1)$ & $76(76.0)$ & & \\
\hline $\mathrm{N}$ & $5(6.1)$ & $8(6.9)$ & $13(13.0)$ & & \\
\hline Location & & & & 0.41 & 0.81 \\
\hline High & $10(11.3)$ & $14(12.7)$ & $24(24.0)$ & & \\
\hline Middle & $18(17.5)$ & $19(19.5)$ & $37(37.0)$ & & \\
\hline Low & $14(13.2)$ & $14(14.8)$ & $28(28.0)$ & & \\
\hline Shape & & & & 5.37 & 0.07 \\
\hline Intraluminal polypoid mass & $19(16.5)$ & $16(18.5)$ & $35(35.0)$ & & \\
\hline Infiltrative mass & $20(24.1)$ & $31(26.9)$ & $5 I(5 I .0)$ & & \\
\hline Bulky & $3(1.4)$ & $0(1.6)$ & $3(3.0)$ & & \\
\hline Unenhanced density & & & & 0.12 & 0.73 \\
\hline Homogeneous & $23(22.2)$ & $24(24.8)$ & $47(47.0)$ & & \\
\hline Heterogeneous & $19(19.8)$ & $23(22.2)$ & $42(42.0)$ & & \\
\hline Enhanced density & & & & 0.02 & 0.90 \\
\hline Homogeneous & $22(21.7)$ & $24(24.3)$ & $46(46.0)$ & & \\
\hline Heterogeneous & $20(20.3)$ & $23(22.7)$ & $43(43.0)$ & & \\
\hline Circumferential extent & & & & 7.31 & 0.06 \\
\hline $\mathrm{CO}-\mathrm{Cl} / 4$ & $2(0.9)$ & $0(I . I)$ & $2(2.0)$ & & \\
\hline $\mathrm{Cl} / 4-\mathrm{Cl} / 2$ & $14(12.3)$ & $12(13.7)$ & $26(26.0)$ & & \\
\hline $\mathrm{Cl} / 2-\mathrm{C} 3 / 4$ & $18(15.6)$ & $15(17.4)$ & $33(33.0)$ & & \\
\hline $\mathrm{C} 3 / 4-\mathrm{Cl}$ & $8(13.2)$ & $20(14.8)$ & $28(28.0)$ & & \\
\hline Boundary of serosa & & & & 0.53 & 0.47 \\
\hline Clear & $13(14.6)$ & $18(16.4)$ & $31(31.0)$ & & \\
\hline Unclear & $29(27.4)$ & $29(30.6)$ & $58(58.0)$ & & \\
\hline Lymph node enlargement & & & & 4.95 & 0.03 \\
\hline Y & II (7.I) & $4(7.9)$ & I5 (I5.0) & & \\
\hline$N$ & $43(39.1)$ & $31(34.9)$ & $74(74.0)$ & & \\
\hline Enhanced lymph node & & & & 0.06 & 0.80 \\
\hline Homogeneous & $34(34.4)$ & $39(38.6)$ & $73(73.0)$ & & \\
\hline Heterogeneous & $8(7.6)$ & $8(8.4)$ & $16(16.0)$ & & \\
\hline Distant metastasis & & & & - & 0.61 \\
\hline Y & $3(2.8)$ & $3(3.2)$ & $6(6.0)$ & & \\
\hline$N$ & $39(39.2)$ & $44(43.8)$ & $83(83.0)$ & & \\
\hline
\end{tabular}

showed that KRAS mutations were associated with $\mathrm{N}$ stage, gross tumor pattern, axial length of the tumor, and the ratio of the axial to the longitudinal dimensions of the tumor on MRI. So far as we know, there is no study that focuses on the correlation between $\mathrm{CT}$ image features and KRAS status of rectal cancer. 
Table 3 Pathological Findings and KRAS Status

\begin{tabular}{|c|c|c|c|c|c|}
\hline & \multicolumn{2}{|c|}{ KRAS Status } & \multirow[t]{2}{*}{ Total } & \multirow[t]{2}{*}{$t / \chi^{2}$} & \multirow[t]{2}{*}{$p$} \\
\hline & Mutated & Wild & & & \\
\hline $\begin{array}{l}\text { Lymphovascular emboli } \\
\text { Y } \\
\text { N }\end{array}$ & $\begin{array}{l}3(3.8) \\
39(38.2)\end{array}$ & $\begin{array}{l}5(4.2) \\
42(42.8)\end{array}$ & $\begin{array}{l}8(8.0) \\
81(81.0)\end{array}$ & - & 0.42 \\
\hline $\begin{array}{l}\text { Signet ring cell } \\
\qquad \\
\mathrm{N}\end{array}$ & $\begin{array}{l}0(0.5) \\
42(4 I .5)\end{array}$ & $\begin{array}{l}\text { I (0.5) } \\
46(46.5)\end{array}$ & $\begin{array}{l}\text { I (I.0) } \\
88(88.0)\end{array}$ & - & 0.53 \\
\hline $\begin{array}{l}\text { Peripheral fat interval infiltration } \\
\text { Y } \\
N\end{array}$ & $\begin{array}{l}23(22.7) \\
19(19.3)\end{array}$ & $\begin{array}{l}25(25.3) \\
22(21.7)\end{array}$ & $\begin{array}{l}48(48.0) \\
4 I(4 I .0)\end{array}$ & 0.02 & 0.88 \\
\hline $\begin{array}{l}\text { Ulcer } \\
\qquad \mathrm{Y} \\
\mathrm{N}\end{array}$ & $\begin{array}{l}19(22.2) \\
23(19.8)\end{array}$ & $\begin{array}{l}28(24.8) \\
19(22.2)\end{array}$ & $\begin{array}{l}47(47.0) \\
42(42.0)\end{array}$ & 1.83 & 0.18 \\
\hline $\begin{array}{l}\text { Lymph node metastasis } \\
\text { Y } \\
\mathrm{N}\end{array}$ & $\begin{array}{l}19(17.0) \\
23(25.0)\end{array}$ & $\begin{array}{l}17(19.0) \\
30(28.0)\end{array}$ & $\begin{array}{l}36(36.0) \\
53(53.0)\end{array}$ & 0.76 & 0.38 \\
\hline $\begin{array}{l}\text { Histological type } \\
\text { Adenocarcinoma } \\
\text { Mucinous adenocarcinoma } \\
\text { Mixed }\end{array}$ & $\begin{array}{l}37(38.7) \\
I(0.9) \\
4(2.4)\end{array}$ & $\begin{array}{l}45(43.3) \\
\text { I ( } 1.1) \\
I(2.6)\end{array}$ & $\begin{array}{l}82(82.0) \\
2(2.0) \\
5(5.0)\end{array}$ & - & 0.32 \\
\hline $\begin{array}{l}\text { Differentiation extent } \\
\text { Low } \\
\text { Middle } \\
\text { High }\end{array}$ & $\begin{array}{l}0(2.8) \\
42(38.7) \\
0(0.5)\end{array}$ & $\begin{array}{l}6(3.2) \\
40(43.3) \\
I(0.5)\end{array}$ & $\begin{array}{l}6(6.0) \\
82(82.0) \\
1(1.0)\end{array}$ & - & 0.03 \\
\hline
\end{tabular}

Note: Fisher's exact method was used.

Our study took in both observed CT features and anatomical-based measurements on preoperative CT images and found that the diameter of the SRV was significantly increased in positive KRAS mutation patients. However, no significant difference was found between the KRAS mutation group and the KRAS wild-type group in the diameter of the IMV. This can be explained by anatomical characteristics, namely that the SRV is the direct vein for the rectum. The key point is that the SRV only receives the blood flow from the rectum, making the SRV particularly sensitive to any factors influencing the biological process of the rectum. However, the IMV receives blood from both the SRV and the sigmoid vein, so there is decreased accuracy of the IMV to predict hemodynamic changes in the rectum.

It is well known that the obstruction of the proximal vein and increased distal blood flow are two major mechanisms for venous dilatation. Our study excluded patients with other neoplastic, infectious, or vascular diseases to ensure the specificity of the SRV predicting blood flow change in the rectum. Earlier research has reported the close relationship between the tumor evasiveness of colorectal cancer and its drainage vein diameters. $\mathrm{Wu}$ et $\mathrm{al}^{12}$ found that rectal cancer patients with positive lymphovascular invasion showed a significantly increased mean superior hemorrhoidal vein diameter. The study by Khan et al further supported this finding, where patients with right hemicolon cancer showed a significantly increased mean SMV (superior mesenteric vein) diameter at presentation. ${ }^{23}$ Therefore, drainage vein diameters may become an effective predictor for the evaluation of colorectal cancer. In our study, we further investigated the possibility of SRV diameter for predicting KRAS mutations in rectal cancer.

Pathologically, increased venous blood flow and emerging collateral vessels in rectal cancer are two leading reasons for the increased diameter of the SRV. The destruction of microcirculation in the tumor increases 
venous blood in the vein, and demand for increased blood supply promotes the development of collateral vessels. VEGF is found to be the strongest mitogen of endothelial cells, which is a key regulator of tumor angiogenesis, vascular remodeling, and vascular sprouting. Vega-Avila and Pugsley ${ }^{24}$ found that VEGF helped vascular proliferation during cancer development, leading to increased blood supply to the tumor. Previous research has shown that KRAS mutations support the production of VEGF and the decline of angiogenesis inhibitor thrombospondin. ${ }^{25-27}$ According to research by Yeo et $\mathrm{al}^{11}{ }^{11}$ the microvessel density evaluated on dynamic contrast-enhanced MRI correlates with the expression level of VEGF, which is consistent with the KRAS mutation. ${ }^{25}$ Also, the study carried out by Krajnovic et $\mathrm{al}^{28}$ found that the simultaneous presence of KRAS mutations and high VEGF expression was related to worse responses to chemoradiotherapy, the frequent appearance of local recurrences, distant metastasis, and shorter overall survival in rectal cancer.

In our study, LNE was also more likely to occur in rectal cancer patients with KRAS mutations. This could be explained by two mechanisms. One is that at the same time as there is increased blood flow of the rectum, lymphatic system homeostasis is maintained by lymphangiogenesis, offering more chances for tumor cells to spread. The other explanation is lymph node metastases. However, according to the pathological results, there was no significant difference in lymph node metastases between the KRAS mutation and wildtype groups. This suggests that hemodynamic factors might have an earlier influence on the enlargement of the lymph node.

In our pathological findings, there was no significant difference between the KRAS mutation group and the KRAS wild-type group in the presence or absence of lymphovascular emboli, signet ring cell, or histological type. This should be interpreted with caution due to the small number of patients with evidence of lymphovascular emboli signet ring cell, or histological type, which were three, one, and five patients, respectively. With regard to differentiation extent $(p=0.03)$, considering the case number of high and low differentiation groups, further research is still needed. In our study, there was no significant difference between the KRAS mutation group and the KRAS wild-type group in the presence and absence of peripheral fat interval infiltration. This might be explained by the fact that local tumor aggressiveness is driven by multiple oncogenes, among which
KRAS was not the only determining factor. ${ }^{29}$ It has been suggested in a study conducted by $\mathrm{Li}$ et $\mathrm{al}^{30}$ that poor tumor cellularity, tumor heterogeneity, and adjuvant therapy may confound the molecular diagnosis of CRC and should be highlighted in prospective assessment. In the process of diagnosis, imaging characteristics could show other foci beside the rectum and provide complementary information to gene testing. There are still many unanswered questions about the association between the mutation at gene level and medical imaging parameters that we can measure. Future studies are still required to explore these possibilities. In medical imaging aspects, MSCT, MRI, and ${ }^{18}$ F-FDG-PET/CT are all important examinations for us to develop further insight into the role of KRAS mutations in rectal carcinoma. Positive results have already been seen on $\mathrm{MRI}^{5,11,22}$ and ${ }^{18}$ F-FDG-PET/CT ${ }^{10,20}$ for predicting KRAS mutations in colorectal carcinoma. However, CT predictors of KRAS mutations in rectal carcinoma have not been described previously. In this study, the increased diameter of the SRV and LNE were found in the KRAS mutation group.

However, there are some limitations to this study. First, LOT was measured on a single plane, with unavoidable factors, including the corrugation and crooked distribution of the rectal wall. Second, though SRV diameter was proven statistically different between the KRAS mutation group and the KRAS wild-type group in our study., the specificity and positive and negative predictive values were unsatisfactory. It might be that due to the relatively small size of SRV diameter, measuring error cannot be avoided in this study. Furthermore, the number of cases in this study was relatively small and the enrolled patients are usually nonadvanced patients according to our inclusion criteria. The selection bias cannot be avoided. Consequently, a larger multicenter sample study is still needed for further research before it can be applied to daily practice. On the other hand, measurement of the SRV diameter with the use of artificial intelligence may be a more advanced method.

\section{Conclusion}

This study has demonstrated feasibility in using the diameter of the SRV to predict KRAS mutations in rectal cancer patients, and LNE on preoperative CT images can also be an important indicator. 


\section{Abbreviations}

KRAS, Kirsten rat sarcoma viral oncogene homologue; MSCT, multi-slice computed tomography; LNE, lymph node enlargement; SRV, superior rectal vein; IMV, inferior mesenteric vein; ER, enhanced ratio; LOT, length of tumor; CRC, colorectal cancer; EGFR, epidermal growth factor receptor; VEGF, vascular endothelial growth factor; AUC, area under the curve; ROC, receiver operating characteristic.

\section{Data Sharing Statement}

All the data concerning this study are available from the corresponding author (Shi-Ting Feng, fengsht@mail.sysu. edu.cn).

\section{Ethics Statement}

The study protocol was approved by the Institutional Review Board of the First Affiliated Hospital of Sun YatSen University and informed consent was obtained from each participant.

\section{Funding}

The National Natural Science Foundation of China $(81771908,81571750,81971684)$, and the Natural Science Foundation of Guangdong Province (2015A030313043).

\section{Disclosure}

The authors declare that they have no competing interests for this work. Chenyu Song; Bingqi Shen; Zhi Dong (equal contribution, co-first author) neither the team of authors (additions or deletions) nor their order of appearance can be changed after submission.

\section{References}

1. Arnold M, Sierra MS, Laversanne M, Soerjomataram I, Jemal A, Bray F. Global patterns and trends in colorectal cancer incidence and mortality. Gut. 2017;66(4):683. doi:10.1136/gutjnl-2015-310912

2. Migliore L, Migheli F, Spisni R, Coppedè F. Genetics, cytogenetics, and epigenetics of colorectal cancer. J Biomed Biotechnol. 2011;2011.

3. Chen W, Zheng R, Baade PD, et al. Cancer statistics in China, 2015. CA Cancer J Clin. 2016;66(2):115-132.

4. Li H-L, Gao Y-T, Zheng Y, et al. [Incidence trends of colorectal cancer in urban Shanghai, 1973-2005]. Chinese journal of preventive medicine. 2009;43(10):875-879.

5. Shin YR, Kim KA, Im S, Hwang SS, Kim K. Prediction of KRAS mutation in rectal cancer using MRI. Anticancer Res. 2016;36 (9):4799-4804. doi:10.21873/anticanres.11039

6. Benson AB, Venook AP, Cederquist L, et al. Colon cancer, version 1.2017, NCCN clinical practice guidelines in oncology. J Natl Compr Canc Netw. 2017;15(3):370-398. doi:10.6004/jnccn.2017.0036
7. Allegra CJ, Jessup JM, Somerfield MR, et al. American Society of Clinical Oncology provisional clinical opinion: testing for KRAS gene mutations in patients with metastatic colorectal carcinoma to predict response to anti-epidermal growth factor receptor monoclonal antibody therapy. $J$ Clin Oncol. 2009;27(12):2091-2096. doi:10.1200/JCO.2009.21.9170

8. Jahn SW, Winter G, Stacher E, et al. Multiple intratumoral KRAS mutations can clonally segregate to different lymph node metastases in colon cancer. Histopathology. 2011;59(2):342-345. doi:10.1111/ j.1365-2559.2011.03902.x

9. Kawada K, Toda K, Nakamoto Y, et al. Relationship between 18F-FDG PET/CT scans and KRAS mutations in metastatic colorectal cancer. J Nucl Med. 2015;56(9):1322-1327. doi:10.2967/ jnumed.115.160614

10. Miles KA, Ganeshan B, Rodriguez-Justo M, et al. Multifunctional imaging signature for V-KI-RAS2 Kirsten rat sarcoma viral oncogene homolog (KRAS) mutations in colorectal cancer. J Nucl Med. 2014;55(3):386-391. doi:10.2967/jnumed.113.120485

11. Yeo DM, Oh SN, Jung CK, et al. Correlation of dynamic contrastenhanced MRI perfusion parameters with angiogenesis and biologic aggressiveness of rectal cancer: preliminary results. J Magn Reson Imaging. 2015;41(2):474-480. doi:10.1002/jmri.24541

12. Wu -C-C, Lee R-C, Chang C-Y. Prediction of lymphovascular invasion in rectal cancer by preoperative CT. Am J Roentgenol. 2013;201 (5):985-992. doi:10.2214/AJR.12.9657

13. Kim JH, Beets GL, Kim M-J, Kessels AGH, Beets-Tan RGH. Highresolution $\mathrm{MR}$ imaging for nodal staging in rectal cancer: are there any criteria in addition to the size? Eur J Radiol. 2004;52(1):78-83. doi:10.1016/j.ejrad.2003.12.005

14. Weiser MR. AJCC 8th edition: colorectal cancer. Ann Surg Oncol. 2018;25(6):1454-1455. doi:10.1245/s10434-018-6462-1

15. Keedy VL, Temin S, Somerfield MR, et al. American Society of Clinical Oncology provisional clinical opinion: epidermal growth factor receptor (EGFR) mutation testing for patients with advanced non-small-cell lung cancer considering first-line EGFR tyrosine kinase inhibitor therapy. J Clin Oncol. 2011;29(15):2121-2127. doi:10.1200/JCO.2010.31.8923

16. Brand TM, Wheeler DL. KRAS mutant colorectal tumors: past and present. Small GTPases. 2012;3(1):34-39. doi:10.4161/ sgtp. 18751

17. Ganeshan B, Mandeville H, Burke M, et al.CT of non-small cell lung cancer (NSCLC): histopathological correlates for texture parameters. Chicago, USA: Radiological Society of North America (RSNA); 2010.

18. Ganeshan B, Miles KA, Young RC, Chatwin CR. In search of biologic correlates for liver texture on portal-phase CT. Acad Radiol. 2007;14(9):1058-1068. doi:10.1016/j.acra.2007.05.023

19. Singh D, Miles K. Multiparametric PET/CT in oncology. Cancer Imaging. 2012;12(2):336. doi:10.1102/1470-7330.2012.9007

20. Lovinfosse P, Koopmansch B, Lambert F, et al. 18F-FDG PET/CT imaging in rectal cancer: relationship with the RAS mutational status. Br J Radiol. 2016;89(1063):20160212.

21. Kawada K, Nakamoto Y, Kawada M, et al. Relationship between 18F-fluorodeoxyglucose accumulation and KRAS/BRAF mutations in colorectal cancer. Clin Cancer Res. 2012;18(6):1696-1703. doi:10.1158/1078-0432.CCR-11-1909

22. Xu Y, Xu Q, Sun H, Liu T, Shi K, Wang W. Could IVIM and ADC help in predicting the KRAS status in patients with rectal cancer? Eur Radiol. 2018;28(7):3059-3065. doi:10.1007/s00330-018-5329-y

23. Khan AN, Botchu R, Patel R, Elabassy M. Dilated SMV in colon cancer-is there any significance. J Gastrointest Cancer. 2012;43 (2):288-295

24. Vega-Avila E, Pugsley MK. An overview of colorimetric assay methods used to assess survival or proliferation of mammalian cells. Paper presented at: Proc West Pharmacol Soc; 2011. 
25. Volpert OV, Dameron KM, Bouck N. Sequential development of an angiogenic phenotype by human fibroblasts progressing to tumorigenicity. Oncogene. 1997;14(12):1495-1502. doi:10.1038/sj. onc. 1200977

26. Rak J, Mitsuhashi Y, Bayko L, et al. Mutant ras oncogenes upregulate VEGF/VPF expression: implications for induction and inhibition of tumor angiogenesis. Cancer Res. 1995;55(20):4575-4580.

27. Figueras A, Arbos MA, Quiles MT, Viñals F, Germà JR, Capellà G. The impact of KRAS mutations on VEGF-A production and tumour vascular network. BMC Cancer. 2013;13(1):125.

28. Krajnović M, Marković B, Knežević-Ušaj S, et al. Locally advanced rectal cancers with simultaneous occurrence of KRAS mutation and high VEGF expression show invasive characteristics. Pathol Res Pract. 2016;212(7):598-603. doi:10.1016/j.prp.2016.02.018
29. Steinestel K, Lennerz JK, Eder S, Kraft K, Arndt A. Invasion pattern and histologic features of tumor aggressiveness correlate with MMR protein expression, but are independent of activating KRAS and BRAF mutations in CRC. Virchows Archiv. 2014;465(2):155-163. doi:10.1007/s00428-014-1604-8

30. Li W, Qiu T, Guo L, Ying J. Major challenges related to tumor biological characteristics in accurate mutation detection of colorectal cancer by next-generation sequencing. Cancer Lett. 2017;410:92-99. doi:10.1016/j.canlet.2017.09.014

\section{Publish your work in this journal}

Cancer Management and Research is an international, peer-reviewed open access journal focusing on cancer research and the optimal use of preventative and integrated treatment interventions to achieve improved outcomes, enhanced survival and quality of life for the cancer patient.
The manuscript management system is completely online and includes a very quick and fair peer-review system, which is all easy to use. Visit http://www.dovepress.com/testimonials.php to read real quotes from published authors. 